\title{
Complexity Analysis of Industrial Organizations Based on a Perspective of Systems Engineering Analysts
}

\author{
I. H. Garbie* and A. A. Shikdar \\ Department of Mechanical and Industrial Engineering, College of Engineering, Sultan Qaboos University, P.O. Box 33, \\ Postal Code 123, Al Khoud, Muscat, Oman
}

Received 4 April 2011; accepted 18 September 2011

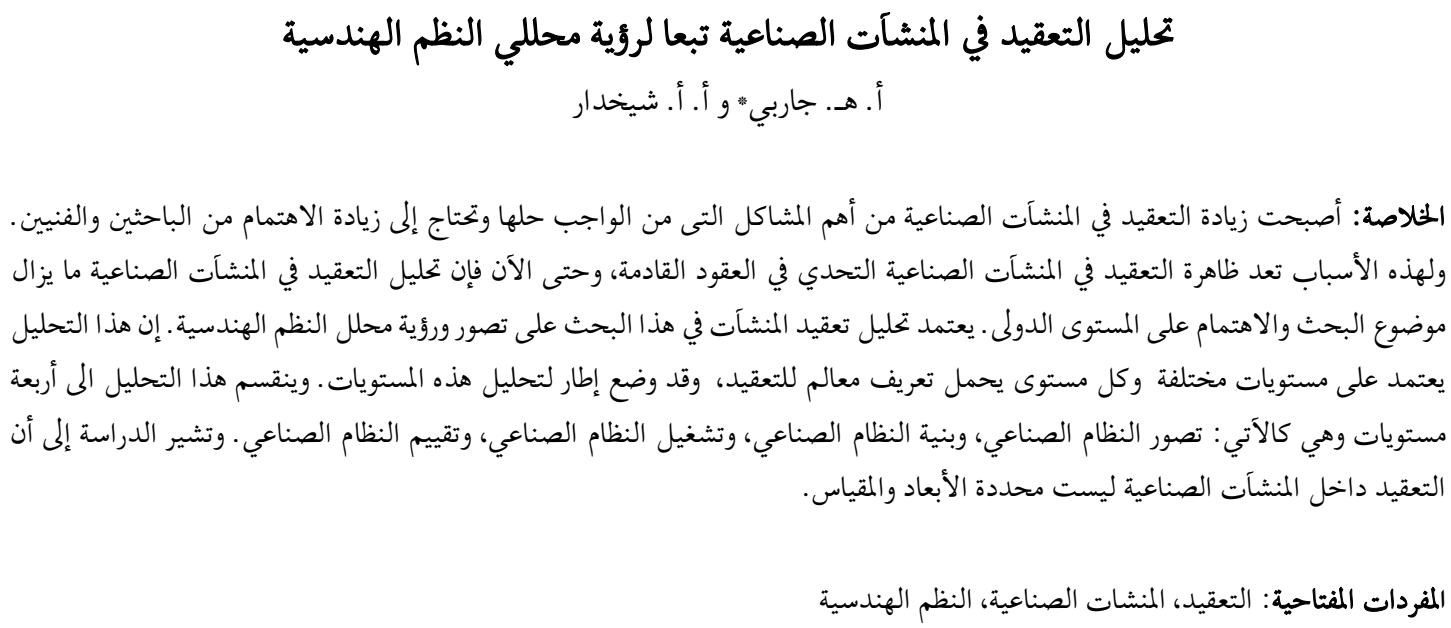

\begin{abstract}
Complexity in industrial organizations became more difficult and complex to be solved and it needs more attention from academicians and technicians. For these reasons, complexity in industrial organizations represents a new challenge in the next decades. Until now, analysis of industrial organizations complexity is still remaining a research topic of immense international interest and they require reduction in their complexity. In this paper, analysis of complexity in industrial organizations is shown based on the perspective of systems engineering analyst. In this perspective, analysis of complexity was divided into different levels and these levels were defined as complexity levels. A framework of analyzing these levels was proposed and suggested based on the complexity in industrial organizations. This analysis was divided into four main issues: industrial system vision, industrial system structure, industrial system operating, and industrial system evaluating. This analysis shows that the complexity of industrial organizations is still an ill-structured and a multi-dimensional problem.
\end{abstract}

Keywords: Complexity, Industrial organization, Systems engineering

\section{Introduction}

Nowadays, industrial organizations complexity exists as an immense international interest and knowledge for the scientific basis. Studying the importance of reducing complexity into industrial organizations was recommended as one of several solutions to

\footnotetext{
*Corresponding author's e-mail:garbie@squ.edu.om
}

recovery the existing financial recession (Garbie 2009 and 2010; Garbie and Shikdar 2009, 2010 and 2011). It was defined as systemic characteristics which integrate several key dimensions of the industrial environment including size, variety, information, uncertainty, control, cost, and value (Kamrani and Adat 2008). Flexibility and agility are considered as the most desir- 
ability of certain system properties for the industrial organizations. These properties will give industrial enterprises more ability to cope with increased environmental uncertainty and adapting to the faster pace of change of today's markets (Giabchetti et al. 2003). The industrial organizations are sometimes viewed as an intrinsic structural property of the system. The structural property is defined as how individual system components relate to each other and how the relationship determines overall system behavior (Arteta and Giabchetti, 2004). Sometimes, manufacturing systems complexity considers the product variety. This means increasing in the product variety increases in the complexity in the manufacturing systems (Kuzgunkaya and ElMaraghy 2006). Also there is another classification of industrial organizations complexity: time-independent complexity and time-dependent complexity. Time -independent complexity is used to add the complexity arising from the designer's perception while time-dependent complexity is either combinational or periodic (Kuzgunkaya and ElMaraghy 2006).

There are several concepts such as: product, process, and operation complexity can be used as a component in industrial enterprises. The product complexity focuses on product features and specifications while the process complexity analysis focuses on the tools, equipment and operations used to manufacture it (Hu et al. 2008). Supply chain management complexity such as: upstream complexity, internal manufacturing complexity, and downstream complexity are considered as complexity issues regarding manufacturing systems (Bazarth et al. 2009). Manufacturing strategy also plays an important role in complexity in industrial enterprise such as Just-in-time manufacturing (lean manufacturing), flexible manufacturing, cellular manufacturing, agile manufacturing, concurrent engineering, etc. The complexity measures are defined as not only intrinsic to the system being studied but also depend on extrinsic properties of the observer. Although most measurements were concentrated on operational measures, both structural and operational characteristics are important to the performance of the system as a whole. Operational complexity measures the uncertainty associated with the material and information flows of the system (Arteta and Giabchetti 2004). The complexity levels in industrial firms are estimated through several case studies based on general framework which includes a questionnaire focusing on each issue in a firm (Garbie and Shikdar 2010).

This paper is organized into several sections. Section 1 presents the importance of complexity concept. Section 2 reviews previous research work about the complexity. Analysis issues are explained in section 3. Section 4 presents a hypothetical example. Conclusions and recommendations for further work will be introduced in Section 5.

\section{Literature Review}

Several research works have been published in this area but none of work mentioned the concept of design for complexity. A methodology based on a simulation model to analyze the complexity in mixed-model assembly production systems was suggested by (Kamrani and Adat 2008). A measurement framework to analyze the structural properties of the enterprise system was presented (Giabchetti et al. 2003). A complexity measure was developed for the business process level inside the organization on one product (e.g. the prepaid phone card) (Arteta and Giachetti, 2004). A new metrics for assessing the structural complexity of system configurations was suggested (Kuzgunkaya and ElMaraghy 2006). They based on machine complexity, buffer type complexity, and material handling system complexity as a structural complexity although they can be considered as an operational or dynamical complexity. Product, process, and operational complexity in modeling and assessment of manufacturing complexity were introduced (ElMaraghy and Urbanic 2003, 2004; ElMaraghy et al. 2005). Each assessment was evaluated independently.

Three different types of complexity were suggested (Bazarth et al. 2009) to represent and model supply chain complexity such as: upstream complexity, internal manufacturing complexity, and downstream complexity. They used these complexities to study the impact effect on a manufacturing plant performance. A coding system of machines, buffers, and material handling equipment to measure complexity based on time-independent complexity (static or structural complexity) of those major components was classified and designed by (ElMaraghy et al. 2005). The structural (static) complexity measure to evaluate the complexity in mixed assembly lines was used (Hu et al. 2008). Several case studies involving 14 Italian companies were conducted (Perona and Miragliotta 2004) to investigate how complexity can affect a manufacturing company's performance. An analytical model for measuring system complexity was presented based on information entropy and probability distribution of resource allocations (Cho et al. 2009). Operational complexity was measured as a function of cost through supply chain management systems (Wu et al. 2007). They indicated that inventory costs are associated with operational complexity. An entropic related to complexity measures to quantify the complexity associated with information content of schedules and variations between schedules was used (Huatuco et al. 2009).

Yang (2010) presented a computational approach to investigate effecting of scheduling with processing 


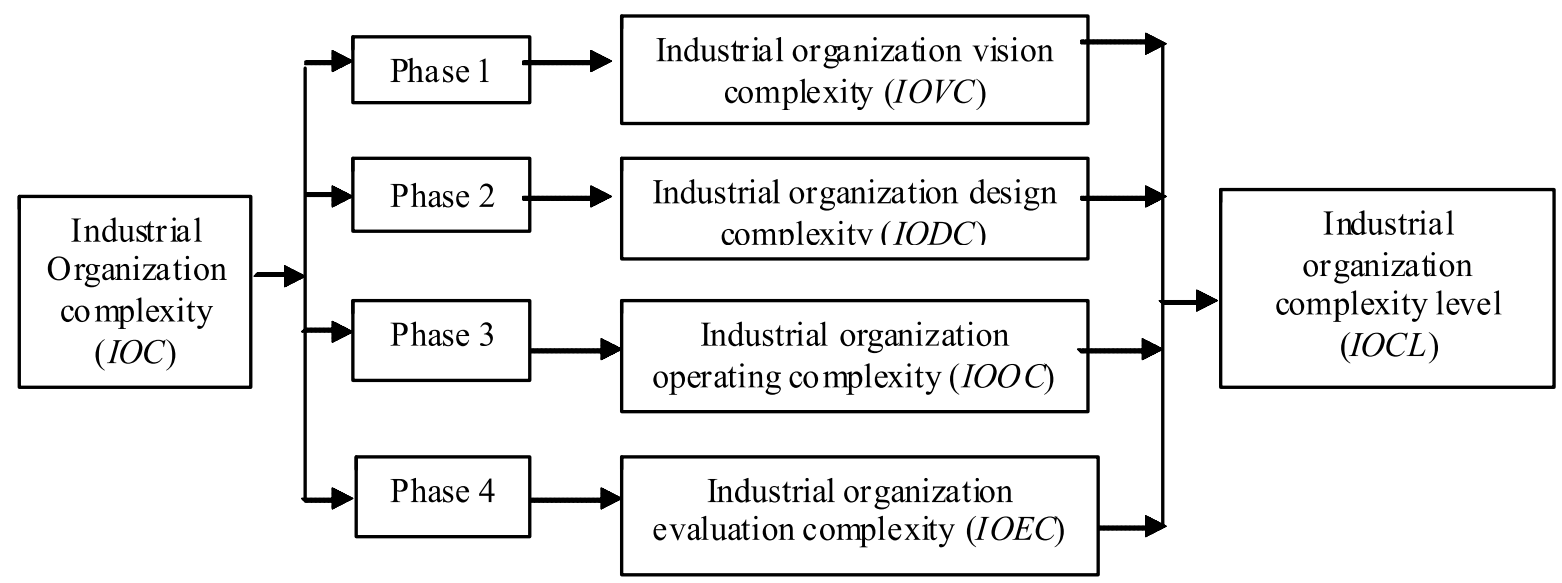

Figure 1. Four phases for design for industrial enterprises complexity

time on two stage hybrid flow shop systems. A mathematical model was suggested to identify functional requirements and the associated design parameters regarding complexity level (Tomiyama et al. 2007). Kashyap and Sinha (2011) studied the complexity related to the mental fatigue required of a person for doing a specific job. While they used how to manage stress, they developed a general model to estimate overall complexity of a profession although they tried to evaluate the job complexity of an engineer. Mazur and Chen (2011) presented organizing work, communication and managing conflict as the most important issues among team members to complete the project. They considered multifunctional knowledge, teamwork capabilities and working relationships with organizing work as a complex problem.

\section{Analysis Issues}

To identify the main issues of industrial organizations complexity $(I O C)$, there are five important questions to be asked such as the following to describe how the complexity of industrial enterprises can be studied. These questions were presented by (Garbie and Shikdar 2011) in analyzing and estimating the complexity levels in industrial organizations.

1. How the complexity issues of an industrial organization is identified and analyzed?

2. How the complexity level of an industrial organization is estimated?

3. How can an industrial organization reduce its complexity?

4. Which issues are more important than others?

5. How can industrial organizations identify the adverse factors for reducing complexity?

Based on these questions, full analysis of complexity regarding these issues will be analyzed and explained through the recommended issues from perspective of systems engineering analysts. These can be represented into four main phases as follows (Fig. 1). Figure 1 shows a briefly industrial organizations complexity procedure consisting of four phases. Each phase will be discussed with its associated issues. It can be noticed from Fig. 1 that the procedure of analyzing industrial organizations complexity follows the four phases parallel.

Based on these concepts and issues mentioned in the previous sections, it can be noticed that the industrial organization complexity (IOC) consists of major issues. These issues are: industrial organization vision complexity $(I O V C)$, industrial organization design complexity $(I O D C)$, industrial organization operating complexity (IOOC), and industrial organization evaluating complexity (IOEC) (as shown in Fig. 1). The mathematical model of industrial organization components and the corresponding complexity relationships between them in order to emphasize on particular vision, design, operating, and evaluating is presented in the following equations ( 1 and 2 ). As each component or element in these systems is a potential source of uncertainty (due to its state), the measuring of complexity for each one is highly valuable. Then, IOCL is clearly modeled as the following Eq. (1) as a function of previous sub-complexities. Eq. (1) can be rewritten as Eq. (2). Each term represents sub-complexity measure of complexity measure of industrial organization $(I O C L)$. Adding these terms with relative weights is considered. These weights can be used as a reason existing to differentiate between major issues of complexity.

$I O C L=f(I O V C, I O D C, I O O C, I O E C)$

$I O C L=w_{I O V C}[I O V C]+w_{I O D C}+w_{I O O C}[I O O C]+$ $w_{\text {IOEC }}[$ IOEC $]$ 
Where:

$I O C L=$ industrial organization complexity level, $I O V C=$ industrial organization vision complexity, $I O D C=$ industrial organization design complexity, $I O O C=$ industrial organization operating complexity, $I O E C=$ industrial organization evaluation complexity.

$w_{\text {IOVC }}, w_{\text {IODC }}, w_{\text {IOOC }}$, and $w_{\text {IOEC }}$ are relative weights of organization vision, organization structure, organization operating, and organization evaluation, respectively. Because the trade-offs frequently exist between these objectives, a comprehensive analysis for each individual measure is needed. The value of these relative weights may reflect the system analyst's subjective preferences based on his/her experience or can be estimated using tools such as Analytical Hierarchy Process $(A H P)$. In this paper, the relative weights using the $A H P$ are estimated and changed frequently according to the new circumstances by decision maker or a group of decision makers (Abdi 2006). These groups are represented in senior management level, manufacturing and/or production engineers, plant managers, operators, and suppliers. These relative weights can be estimated using $A H P$ according to the next matrix. For example, suppose, then this means that industrial organization design complexity $(I O D C)$ is four times more important than operating complexity $(I O O C)$.

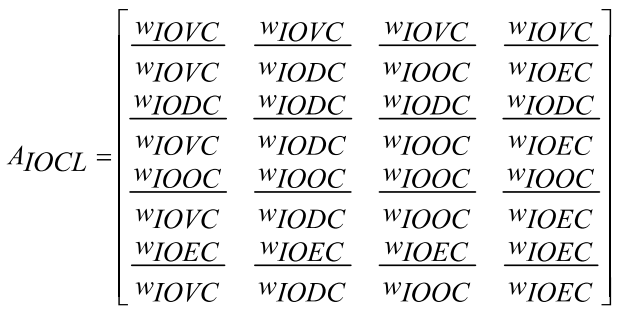

\subsection{Phase 1: Industrial Organizations Vision Complexity}

The industrial organizations vision complexity is the first step in the design for industrial enterprises complexity. The major issue of this phase is how to collect the main components (elements) of industrial organizations vision. The organization vision usually specifies what supply chain management (SCM) representing in number of suppliers $(N O S)$, demand variability $(D V)$ representing in number of customers $(N O C)$, introducing s new product $(N P)$, product life cycle $(P L C)$ representing also in product development $(P D)$, and time to market (TTM) requires and how they are affected and effecting on the complexity of industrial organizations. The industrial organizations vision complexity $(I E V C)$ will be represented mathematically as a function of these issues as shown in the following Eq. (3).
$I E V C=f(S C M(N O S), D V(N O C), N P, P L C$

(PD), TTM)

\subsection{Phase 2: Industrial Organizations Design Complexity}

The second phase, industrial organizations design complexity (IODC) procedure is the designing for system complexity itself. It is mainly concerned with different elements to represent the complexity of it. These elements are: product structure and design (PSD), system design $(S D)$, and manufacturing strategies $(M S)$. For each main element, there are several sub-main elements which play an important role in the value of complexity. For example, the PSD has four different types to represent the complexity in the product design such as number of parts per product $(N N P)$, number of operations per part $(N O P)$, processing or manufacturing time per operation $(P T)$, product size and weight $(P S W)$. All of them have a significant effect on the complexity of manufacturing/production process. System design $(S D)$ is playing a major role in complexity in industrial organizations. It can be observed how complexity is the analysis of the system design $(S D)$. The $S D$ divides the complexity analysis into three major issues: production system size (PSS), material handling system $(M H S)$, and plant layout system $(P L S)$. For the PSS, there are three different classifications of production system: small-sized production system (SSPS), medium-sized production system (MSPS), and large-sized production system (LSPS). Each classification type represents or introduces a significant effect on complexity.

Also, the material handling system (MHS) and plant layout system $(P L S)$ play an important role in identifying the complexity in industrial organizations. The $M H S$ consists of material handling equipments $(M H E)$ with different types of equipments (e.g. conveyor, trucks, forklifts, crane, etc.), material handling storage system (MHSS) (e.g. manual or automated storage and retrieval), and identification systems (IS). How it can be thought about all previous components and degree of complexity related to each one. Facility planning or plant layout system $(P L S)$ has a significant effect on complexity by different types of configuration. It can be seem that functional layout $(F L)$ or process layout is more complex than product layout $(P L)$ and/or cellular layout $(C L)$. Complexity with respect to manufacturing strategies $(M S)$ is totally different than $(S D)$ and $(P S D)$ because it was looking for which strategy can be applied. Complexity in lean manufacturing $(L M S)$ for example is affected by increased product variety if it is comparing with product layout $(P L)$ in mass production system. But in general, complexity in lean manufacturing system $(L M S)$ is low comparing it with agile manufacturing system $(A M S)$, flexible man- 
ufacturing system (FMS), and reconfigurable manufacturing system (RMS). Regarding complexity in $A M S, F M S$, and $R M S$, they can deal easily with any changes in product design (modifications), unpredictable demand, etc. but infrastructure of these systems itself is more sophisticated and complicated to lead these systems to be more and more complex.

The mathematical expression of industrial organizations design complexity (IODC) can be modeled as the following Eqs. (4 and 5) in different facets.

$I O D C=f(P S D, S D, M S)$

$I O D C=w_{\mathrm{PSD}}(P S D)+w_{\mathrm{SD}}(S D)+w_{\mathrm{MS}}(M S)$

$I O D C=f(N P P, N O P, P T, P S W, M H S, P S S$, PLS, LMS, FMS, AMS, RMS)

The $w_{\mathrm{PSD}} w_{\mathrm{SD}}$ and $w_{\mathrm{MS}}$ are relative weights of product design, system design and manufacturing strategies, respectively. Eq. (5) can be rewritten as a very board general representing the lowest level of information in industrial organization design complexity as the following Eq. (6).

$$
\begin{aligned}
I O D C= & f(N P P, N O P, P T, P S W, M H E, M H S S, \\
& I S, S S P S, M S P S, L S P S, F L, C L, P L, \\
& L M S, F M S, A M S, R M S)
\end{aligned}
$$

\subsection{Phase 3: Industrial Organizations Operat- ing Complexity}

Once the industrial organization system vision and design complexities become available to the perspective of industrial systems analysts and designers, the design for complexity related to the system operating becomes urgent to be analyzed and evaluated. This phase may involve further activities in data collection and processing. Design for industrial organizations operating or dynamic complexity (IOOC) is different than previous ones (system vision, and system design). In this analysis, it can be noticed that there are three major items of complexity: resource status of operating complexity ( $R S O C$ ), work in progress complexity $(W I P C)$, and business operations complexity $(B O C)$. Resources mean equipment (e.g. machining equipment, forming equipment, material handling equipment, etc.) and human. In this analysis, it will be concentrated on the resource reliability $(R R)$, resource capability or flexibility $(R C)$, resource utilization $(R U)$, resource scheduling/ rescheduling $(R S / R)$, and human scheduling/rescheduling $(H S / R)$. For example, maintenance level plays a vital role in resource reliability. This means that the lower the maintenance level is the lower in machine capacity (reliability). Also, work in progress complexity (WIPC) representing in buffer between workstations or departments is considered one of measuring degree of complexity inside the production plant (factory).
There are several important issues that can be used to evaluate the business operations complexity (BOC). These issues are: organization plans $(O P)$, organizing work $(O W)$, structure of management levels $(S M L)$, staffing developing and motivation $(S D M)$, decision making $(D M)$, communication between and within management levels $(C M L)$, managing conflict, change, culture and stress (MCS), and finally leadership roles in management $(L R)$. Then, industrial organizations operating complexity (IOOC) can be modeled to measure or evaluate the complexity level as the following Eqs. (7 and 8).

$I O O C=f(R S O C, W I P C, B O C)$

$I O O C=w_{\mathrm{RSO}}(R S O)+w_{\mathrm{WIP}}(W I P)+w_{\mathrm{BOC}}(B O C)$

$I O O C=f(R R, R C, R U, R S / R, H S / R, W I P(B S), O P$, $O W, S M L, S D M, D M, C M L, M C S, L R)$

\subsection{Phase 4: Industrial Organizations Evalua- tion Complexity}

The fourth phase in the design for industrial organizations complexity $(I O E C)$ procedure is the complexity regarding the system evaluation. As industrial organizations have a great impact on the performance measurements, they still have a problem in measuring these complexities especially regarding selection of the objectives. In this paper, there are five different objectives that can be used to evaluate the complexity. They are: product cost $(P C)$, response $(R)$ representing in manufacturing lead time, system productivity $(S P)$ representing in system utilization, product quality $(P Q)$ representing in number of scrap (defect rate), and appraising and rewarding performance $(A R P)$. They also can be modeled mathematically as the following Eq. (9).

$I O E C=f(P C, R, S P, P Q, A R P)$

\section{A Hypothetical Example}

This numerical example is used to estimate the level of complexity of industrial organizations. This can be implemented through three sequential steps. The first step is used to estimate the relative weights of industrial organizations based on the four major issues, $I O V C, I O D C, I O O C$, and IOEC. The relative weights between these issues are estimated by using the $A H P$ as the following matrix based on the pair wise comparisons of the four major issues. It can be noticed that the relative weights of $I O V C$ is estimated to be equivalent to the $I O D C$, twice as important as the $I O O C$ and four times more important than the IOEC. The IODC is estimated to be three times more important than the $I E O C$ and four times more important than IOEC. The 
$I O O C$ is also estimated to be twice as important as the IEEC. As a result, the values of relative weights are estimated at $0.35,0.40,0.16$, and 0.09 for $I O V C$, $I O D C$, IOOC, and IOEC, respectively. Eq. (2) is used to calculate the industrial enterprises complexity level (IOCL) incorporating of the four major issues as the following Eq. (10).

$$
\text { AIOCL }=\left[\begin{array}{cccc}
1 & 1 & 2 & 4 \\
1 & 1 & 3 & 4 \\
0.50 & 0.33 & 1 & 2 \\
0.25 & 0.25 & 0.50 & 1
\end{array}\right]
$$

$$
\begin{aligned}
& I O C L=0.35[I O V C]+0.40[I O D C]+0.16 \\
& {[I O O C]+0.09[I O E C]}
\end{aligned}
$$

In the second step, the multiple regression models are used to estimate and formulate the complexity levels for the major and sub-major issues. The values were assumed to follow a uniformly distributed random variable with known parameters as shown as in Table 1 for 50 generating values for each issue. The multiple regression models for industrial organizations vision complexity, industrial organizations design complexity (product structure and design, system design and manufacturing strategies), industrial organizations operating complexity (resource status and business), and industrial organizations evaluation complexity are presented as the following Eqs. (1117), respectively. This was done by using MINITAB statistical software package to generate a random variable and formulating a multiple regression model.

$I E V C=-0.0356-0.0374(N O S)-0.000013(N O C)+$ $0.0065(P D)+0.0842($ TTM $)$

$P S D C=0.359-0.00436(N P P)+0.0353(N O P)+$ $0.0123(P T)+0.0759($ ind 1$)-0.0420($ ind 2$)+0.0241$ (ind 3) - 0.0039 (ind 4)

ind 1 , ind 2 , ind 3 , and ind 4 are used for the product size and weight.

$S D C=0.2440+0.00479(S S P S)-0.00795(M S P S)+$ $0.00018(L S P S)+0.0848(F L)-0.0681(C L)+0.0609$ $(P L)+0.092(M H S S)+0.0290(M H E)+0.189(I S)$

$M S C=0.441-0.059(L M S)+0.245(F M S)-0.028$ $(A M S)-0.024(R M S)$

$R O S C=0.598-0.379(R R)+0.509(R C)+0.113(R U)$ - $0.50(R S / R)+0.233(H S / R)$
$B O C=0.980-0.0029(O P)-0.0076(O W)+0.0015$

$(S M L)+0.171(S)+0.234(D)+0.0050(M)-0.0266$

$(D M)-0.0642(C M L)-0.134(M C S)-0.289(L R)$

IOEC $=0.457+0.00210(P C)+0.0088(R)-0.172$ $(S P)-0.40(P Q)+0.117(A R P)$

The third step is used to determine the complexity level of $I O V C, P S D C, S D C, R O S C$, and $I O E C$, respectively by optimizing Eqs. (11-17). The constraints are identified based on the range values of each sub-issue which are listed in Table 1.

To determine the relative weights between PSDC, $S D C$, and $M S C$ and between $R S O C, W I P C$, and BOC, the following matrices are used to estimate these values and the pair wise comparison between product structure and design, system design and manufacturing strategies is illustrated in these matrices.

$$
\mathrm{A}_{\mathrm{IODC}}=\left[\begin{array}{ccc}
1 & 1 & 2 \\
1 & 1 & 2 \\
0.5 & 0.5 & 1
\end{array}\right] \quad \mathrm{A}_{\mathrm{IOOC}}=\left[\begin{array}{ccc}
1 & 4 & 2 \\
0.25 & 1 & 0.25 \\
0.5 & 4 & 1
\end{array}\right]
$$

With respect to $I O D C$, it can be noticed that a PSDC is estimated to be equivalent to the $S D C$ and twice as important as a $M S C$. The same estimation is done relate to the $S D C$ with $P S D C$ and $M S C$. Regarding the $I O O C$, it can be noticed that $R S O C$ is estimated to be four times more important than the WIPC and two times as important as the $B O C$. The $B O C$ is also estimated to be four times more important than WIPC. As a result, the relative weights for $I O D C$ between $P S D C$, $S D C$, and $M S C$ are estimated at $0.40,0.40$, and 0.20 , respectively. Also, the relative weights for IOOC between $R S O C, W I P C$, and $B O C$ are estimated at $0.544,0.110$, and 0.345 , respectively. The results of complexity level in each sub-issue and major issue are illustrated in Table 2. Then, Eq. (10) is used again to calculate the global complexity level based on the information taken from Table 2 and the estimated relative weights as the following.

$I O C L=0.35[0.4582]+0.40[0.3099]+0.16[0.3023]$ $+0.09[0.4570]=0.3740$

It can be noticed from the results given from the previous equation and Table 3 that the level of complexity in this enterprise equals to $37.40 \%$ and this value seems ranked in a medium range. It seems that vision complexity represents more important $(0.16 / 0.3740=42.78 \%)$ than design complexity $(33.15 \%)$. The percentage values of operating and evaluating complexity are $13.36 \%$ and $10.70 \%$, 
Table 1. Data for complexity issues based on uniform distribution, $U$ [a, b]

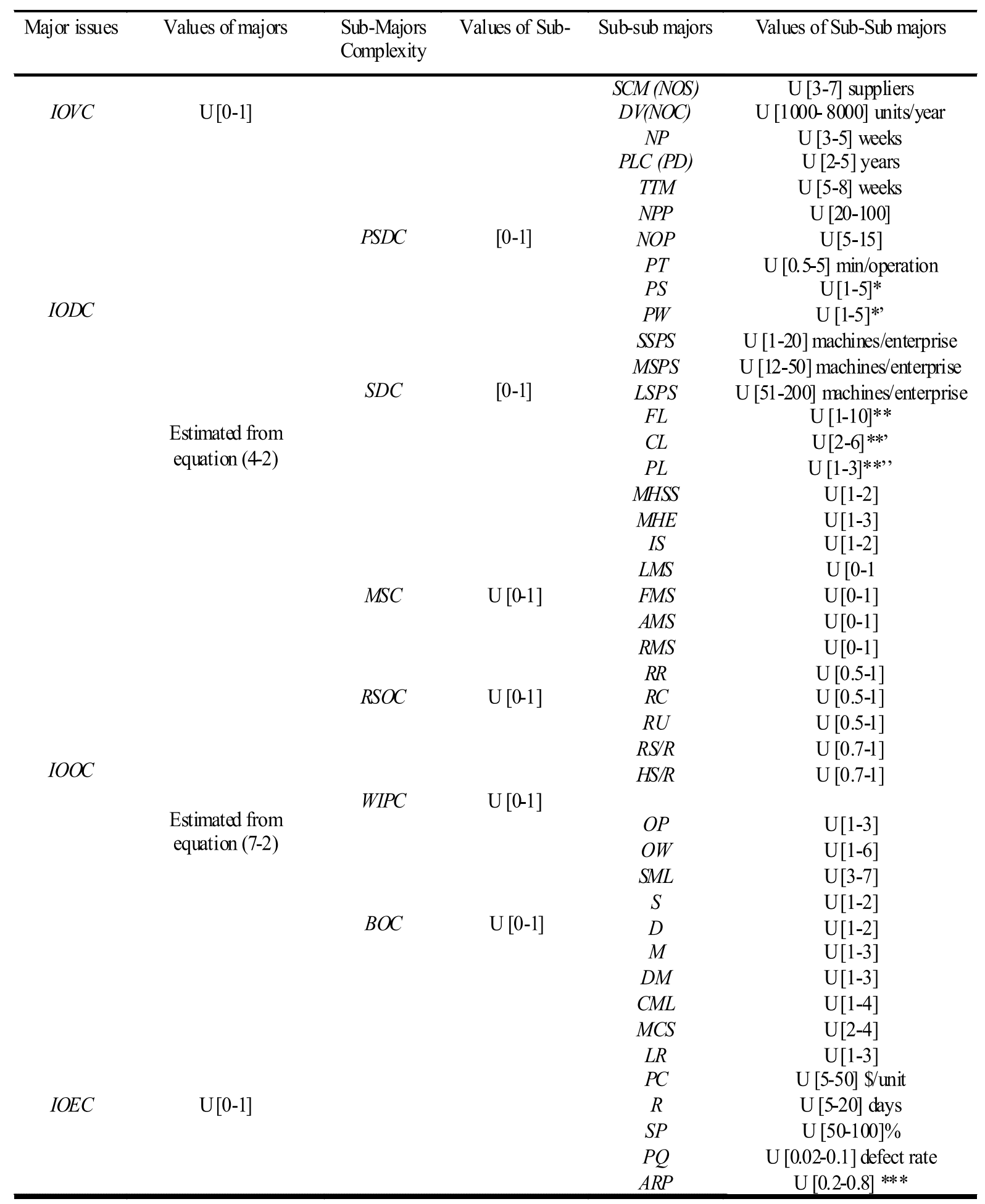

$\mathrm{U}$ represents the uniform distribution with [a] the lower limit and [b] is the upper limit, *represents the product size (small, small-medium, medium, medium-large, large), *'represents the product weight (light, light-medium, medium, medium-heavy, heavy), ${ }^{* *}$ represents the degree of variety in functional layout, ${ }^{* *}$ 'represents the number of manufacturing cells, ${ }^{* *}$ "represents the variety of models (single, batch, and mixed), ${ }^{* * *}$ represents the appraising and rewarding performance as a percentage. 
Table 2. Complexity levels in the four phases of industrial enterprises

\begin{tabular}{cccc}
\hline Major complexity issue & $\begin{array}{c}\text { Complexity } \\
\text { value }\end{array}$ & Sub-major issue & Complexity of Sub-major \\
IOVC & 0.4582 & & 0.3828 \\
& & $P S D C$ & 0.3364 \\
IODC & 0.3099 & $S D C$ & 0.1111 \\
& & $M S C$ & 0.4049 \\
IOOC & RSOC & 0.650 \\
IOEC & 0.3023 & WIPC & 0.030 \\
\hline
\end{tabular}

Table 3. Values of major issues regarding the complexity level

\begin{tabular}{cccc}
\hline $\begin{array}{c}\text { Industrial Organization Complexity } \\
\text { Level (IOCL) }\end{array}$ & $\begin{array}{c}\text { Major } \\
\text { Issues }\end{array}$ & Value of major issues & $\begin{array}{c}\text { Percentage of Major } \\
\text { issues }(\%)\end{array}$ \\
& IOVC & 0.160 & 42.78 \\
0.3740 & IODC & 0.124 & 33.15 \\
& IOOC & 0.050 & 13.36 \\
& IOEC & 0.041 & 10.70 \\
\hline
\end{tabular}

respectively. These values can be totally different from an industrial organization to another one based on the sub-major issues and the relative weights between submajor and major issues. Regarding the complexity reduction, it can be noticed that if the values of submajor issues reduced, the complexity level will be reduced too. It also seems that the vision complexity and design complexity represent the adverse factors for reducing complexity. With respect to IOVC, it is not easily to reduce the number of suppliers, for example, and it should increase this number. Also, for IODC, the number of parts per product, number of operations per part, processing time per operation, product size and weight, production system size are representing adverse factors for reducing complexity because they cannot be changed.

\section{Conclusions}

It can be noticed from this analysis that complexity issues are not simple. It required emphasize on each of the main issues and the sub-main. Hence, industrial organizations complexity (IOC) will involve four major issues: vision complexity, design complexity, operating complexity, and evaluation complexity. Analyzing complexity in industrial enterprises is based on the four main issues and it can be mathematically expressed as a total global function as the following Eq. (18).
$I O C=f(S C M(N O S), D V(N O C), N P, P L C(P D)$, TTM, NNP, NOP, PT, PSW, MHE, MHSS, IS, SSPS, MDPS, SLPS, FL, CL, PL, LMS, FMS, AMS, RMS, RR $(M L), R C<R U, R S / R, H S, / R, W I P(B S), O P, O W$, $S M L, D M, C M L, L R, S D M, M C S, P C, R, S R, P Q$, $A R P)$

The $I O C$ issues should be dynamic and they should evolve with and adapt to the changing internal and external environment. Until now, the $I O C$ remains a research topic of immense international interest. This will represent the degree of freedom of industrial organizations designers to identify which issue is more significant than others. The main contribution in this paper is how to identify and model the components of industrial enterprises complexity in any industrial firms (organizations) at any time considering these components. The authors intend to extend this research to apply this analysis and formulation the suggested model to estimate and optimize the degree of complexity in any industrial enterprises towards full validation of the complexity theory which will be discussed and presented in the future research.

\section{Acknowledgments}

The authors would like to acknowledge the financial support provided by the Sultan Qaboos University (Grant No. IG/ENG/MIED/10/01) to carry out this research work. 


\section{References}

Abdi MR (2006), Performance evaluation of reconfigurable manufacturing. The Proceedings of the $36^{\text {th }}$ CIE Conference on Computer and Industrial Engineering, Taipei, Taiwan 2479-2491.

Arteta BM, Giachetti RE (2004), A measure of agility as the complexity of the enterprise system. Robotics and Computer-Integrated Manufacturing 20:495-503.

Bazarth CC, Warsing DP, Flynm BB, Flynn EJ (2009), The impact of supply chain complexity on manufacturing plant performance J. of Operations Management 27:78-93.

Cho S, Alamoudi R, Asfour S (2009), Interactionbased complexity measures of manufacturing systems using information entropy. Int. J. of Computer Integrated Manufacturing 22(10): 909922.

ElMaraghy WH, Urbanic RJ (2003), Modeling of manufacturing systems complexity. Annals of the CIRP 53(1):363-366

ElMaraghy WH, Urbanic RJ (2004), Assessment of manufacturing complexity. Annals of the CIRP, 53(1):401-406.

ElMaraghy HA, Kuzgunkaga O, Urbanic RJ (2005), Manufacturing systems configuration complexity. Annals of the CIRP 54:445-448.

Garbie IH, Shikdar A (2009), Determination of complexity levels in industrial firms. Proceedings of the Int. Conference of Industrial Engineering Research (IERC), Miami, Florida, USA, May 30June 3:1423-1428.

Garbie IH (2009), A vision for reconfiguring industrial organization due to the global recession. Proceedings of the $39^{\text {th }}$ of Int. Conference on Computers and Industrial Engineering, France, 658-663, July 6-8, 2009.

Garbie IH, Shikdar A (2010), Complexity level in industrial firms: Case studies and implementation. Proceedings of the 2010 International Conference on Industrial Engineering and Operations Management (IEOM 2010), Dhaka, Bangladesh, January 9-10, 2010.

Garbie IH (2010), A roadmap for reconfiguring industrial enterprises as a consequence of global economic crisis (GEC). J. of Service Science and Management 3(4):419-428.
Garbie IH, Shikdar A (2011), Analysis and estimation of complexity level in industrial firms. Int. J. of Industrial and Systems Engineering 8(3):1-23.

Giabchetti RE, Martinez LD, Saenz OA, Chen CS (2003), Analysis of the structural measures of flexibility and agility using a measurement theoretical ramework. Int. J. of Production Economics 86: 47-62.

Hu SJ, Zhu X, Koren WY (2008), Product variety and manufacturing complexity in assembly systems and supply chains. Annals of the CIRP 57: 45-48.

Huatuco LH, Efstathiou J, Calinescu A, Sivadasan S, Kariuki S (2009), Comparing the impact of different rescheduling strategies on the entropic-related complexity of manufacturing systems. Int. J. of Production Research 47(1):4305-4325.

Kamrani AK, Adat A (2008), Manufacturing complexity analysis: A simulation-based methodology-collaboration engineering. Theory and Practice edited by Karmrani AK Springer, 227-248.

Kashyap N, Sinha S (2011), Estimation of job complexity in an industrial organization. Int. J. of Industrial and Systems Engineering Vol. 7(1):2644.

Kuzgunkaya O, ElMaraghy HA (2006), Assessing the structural complexity of manufacturing systems configurations. Int. J. of Flexible Manufacturing Systems 18:145-171.

Mazur LM, Chen SJ (2011), A task-member assignment model for complex engineering. Int. J. of Industrial and Systems Engineering 7(1):1-25.

Perona M, Miragliotta G (2004), Complexity management and supply chain performance assessment. Int. J. of Production Economics 90:03-115.

Tomiyama T, Amelio VD, Urbanic J, ElMaraghy HW (2007), Complexity of multi-disciplinary design. Annals of the CIRP 56(1):85-188.

Wu Y, Frizelle G, Efstathiou J (2007), A study on the cost of operational complexity in customer-supplier systems. Int. J. of Production Economics 106:217-229.

Yang J (2010), A new complexity proof for the twostage hybrid flow shop scheduling problem with dedicated machines. Int. J. of Production Research 48(5):531-1538. 